at Stanford University, California, is to attach a molecule to two beads and use two optical tweezers, neither of which is physically attached to the lab environment, to hold the molecule. Bustamante's group recently added another element to this approach - the use of a single laser beam that splits into the two optical tweezers, to control for variation in laser light by providing feedback on both traps (J. R. Moffitt et al. Proc. Natl Acad. Sci. USA 103, 9006-9011; 2006).

Companies are eyeing the area of optical tweezers seriously. Carl Zeiss in Jena, Germany now offers the Palm Micro Tweezers that use either a red or near infrared laser that can be split into two independent traps. Arryx, in Chicago, Illinois, has taken a different approach for its optical-tweezer technology, called BioRyx 200, which is a holographic optical trap created from a spatial light modulator and allows the control of up to 200 objects simultaneously.

$\mathrm{Ha}$ is starting to combine optical tweezers with single-molecule FRET to study the enzymes involved in transcription and translation.

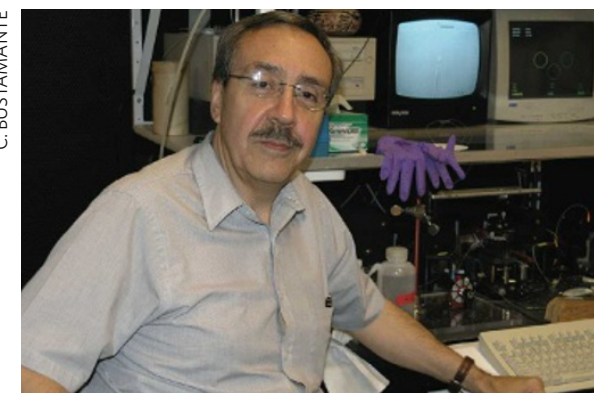

Carlos Bustamante would like to see a wider adoption of techniques to study single molecules.
He thinks that the combination of these techniques is necessary as single-molecule research moves towards studying more complex properties of enzymes and proteins. "It is now possible to measure the fundamental reaction steps of the enzymes using optical traps to obtain single base pair resolution," says $\mathrm{Ha}$, but he adds that using FRET allows conformational changes of the enzymes themselves to be monitored at the same time. This combination allowed Ha's team to directly measure via fluoresence conformational changes as a function of applied force to dissect the landscape of the Holliday junction - a mobile junction between four strands of DNA (S. T. Hohng et al. Science 318, 279-283; 2007).

\section{Added colour}

$\mathrm{Ha}$ is moving into three-colour FRET with optical traps to get more information from each experiment. Whereas two-colour FRET can measure one distance, multiple-colour FRET will provide additional distance information from each experiment. Ha is using three-colour FRET (one donor and two acceptors) to study the diffusion of protein on single-stranded DNA and the enzymatic activity of the ribosome.

Bustamante is also excited about the possibility of using FRET with optical tweezers. "The bottom line is that it will allow you to follow a particular biological process in a multidimensional fashion,"he says. His lab has built a prototype optical trap, called 'minitweezers', that will incorporate fluorescence. With this, the team hopes to bring together the whole optical table into an instrument the size of two

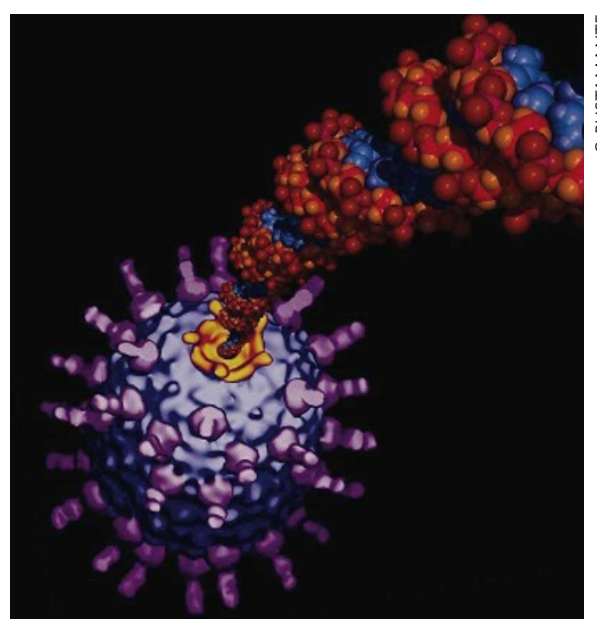

Schematic representation of a molecule attached to a bead within an optical trap.

\section{coffee cans, Bustamante says.}

Measurements of force and interaction at the single-molecule level are being reported with increased frequency. And there is interest from the wider biological community: at the 2007 meeting of the American Society for Cell Biology there was a session focused on the analysis of mechanical forces. "It was pretty amazing to see a ballroom of more than a thousand people listening to talks on the significance of mechanical properties of cells," Tille says. And with developers actively trying to engage biologists while continuing to advance the power of these technologies, one has to wonder how many more will be listening next year. Nathan Blow is technology editor for Nature and Nature Methods.

\title{
SHRINKING DOWN GAS CHROMATOGRAPHY
}

Imagine looking down at your watch to check the time, while the same watch performs chromatography to determine the chemical composition of the air around you. Although unlikely to be in the shops any time soon, recent advances in carbon nanotube technology and surface chemistry could make very small gas-chromatography devices a future reality.

Michael Strano's group at the Massachusetts Institute of Technology in Cambridge has been developing a highly sensitive, miniaturized gaschromatography instrument based on carbon nanotubes. Sensitivity was key for this device, Strano wanted to be able to detect analytes in the part-per-trillion range - an amount so small it can be difficult to grasp at first. "This is like opening a small vial in a crowded high-school gymnasium,"
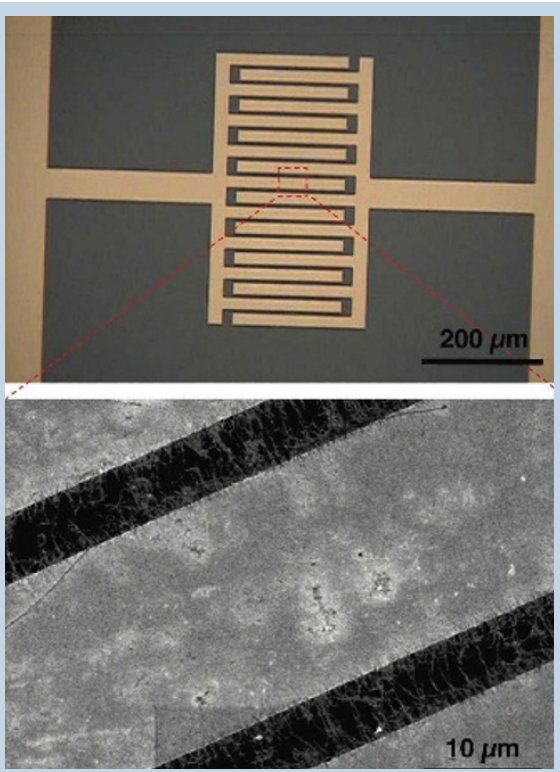

Micro-GC nanotube sensor array (above); close up of a nanotube with array (below).

explains Strano, "even if you open the vial and shut it quickly, you have actually released hundreds of parts per trillion of this mixture everywhere."

For the backbone of its new gas-chromatography instrument, Strano's group created singlewalled nanotube, chemi-resistor arrays in which the nanotubes were aligned between electrodes.

Changes in the electrical resistance can be analysed as a desired molecule binds the nanotube. To tune the binding of the analyte to the arrays the group also had to develop a new chemistry (C. Y. Lee and M. S. Strano J. Am. Chem. Soc. 130, 1766-1773;

2008). "You can use mean basicity to target whether your molecules will bind very strongly, in between, or not at all," explains Strano. Then using a microelectromechanical systems device it should be possible to have precise control over which molecules bind to the array.

As a proof-of-principle, Strano's group created a very simple device by etching a chromatography column on a chip. Using a 100micrometre trench the team showed that mixtures can be separated and, using a fast detector, it is possible to transduce each peak as it comes out.

Strano group's demonstrated parts-per-trillion detection on its device, confirming the potential of this approach to miniaturized, onchip gas chromatography. And for Strano, seeing an actual physical device working at that level was extremely rewarding. “We are engineers, so we try to make devices that work." 